\title{
An Expeditious, Green and Protecting-Group-Free Synthesis of a Potent Secondary Metabolite Calebin-A and Its Analogues
}

\author{
Anju Majeed \\ Kalyanam Nagabhushanam* \\ Muhammed Majeed \\ Samuel Manoharan Thomas \\ Nooruddin Thajuddin \\ SynOpen 2017, 1, 125.
}

The spelling of the last author name has been corrected. 